\title{
Photon-Number Squeezing in Circuit Quantum Electrodynamics
}

\author{
M. Marthaler ${ }^{1}$, Gerd Schön ${ }^{1}$, and Alexander Shnirman ${ }^{2}$ \\ ${ }^{1}$ Institut für Theoretische Festkörperphysik and DFG-Center for Functional Nanostructures (CFN), \\ Universität Karlsruhe, D-76128 Karlsruhe, Germany \\ ${ }^{2}$ Institut für Theoretische Physik, Universität Innsbruck, A-6020 Innsbruck, Austria
}

(Dated: February 5, 2008)

\begin{abstract}
A superconducting single-electron transistor (SSET) coupled to an anharmonic oscillator, e.g., a Josephson junction- $L-C$ circuit, can drive the latter to a nonequilibrium photon number state. By biasing the SSET in a regime where the current is carried by a combination of inelastic quasiparticle tunneling and coherent Cooper-pair tunneling (Josephson quasiparticle cycle), cooling of the oscillator as well as a laser like enhancement of the photon number can be achieved. Here we show, that the cut-off in the quasiparticle tunneling rate due to the superconducting gap, in combination with the anharmonicity of the oscillator, may create strongly squeezed photon number distributions. For low dissipation in the oscillator nearly pure Fock states can be produced.
\end{abstract}

PACS numbers: 74.50.+r 05.45.-a 05.60.Gg 33.80.Wz

In driven oscillator systems, depending on the type of driving, different nonequilibrium photon (or phonon) populations can be produced, and indeed for many applications the production of specific photon number distributions is crucial. Quantum cryptography and linear optical quantum computation require a reliable supply of single photons [1, 2], whereas for quantum measurements it may be of advantage to use strongly squeezed photon distributions 3. A well-known optical system where strongly squeezed states can be created is the micromaser [4. In this article we describe how highly squeezed photon (phonon) number distributions can be produced in suitable superconducting quantum circuits.

Recent experiments with such circuits, with superconducting qubits coupled to electromagnetic resonators ("circuit QED"), confirmed various concepts developed in the field of cavity QED but also provided important extensions, e.g., to the regime of strong coupling [5, 6, 7. 8, 9. A Josephson qubit, ac-driven to perform Rabi oscillations in resonance with an oscillator, depending on the detuning either cools the oscillator or produces a laser-like enhancement of the photon numbers [10, 11. Similarly, a superconducting SET (SSET) biased at the Josephson quasiparticle (JQP) cycle can be used to cool or drive an oscillator [12, 13, 14, 15. Squeezing of the photon number distribution has been predicted, but as described in the literature it is only a weak effect [11, 16]. Here we show that by exploiting the gap structure of the quasiparticle tunneling rate in combination with an anharmonicity of the oscillator, strongly squeezed photon number states, close to a pure Fock state can be produced (see fig. 1).

The system to be studied consists of a SSET coupled to an anharmonic superconducting or nanomechanical oscillator (see fig. 2p. The SSET is formed by a superconducting island coupled via low-capacitance tunnel junctions to two superconducting leads. A gate voltage $V_{\mathrm{G}}$ shifts the electrostatic energy of the island and controls, together with the transport voltage $V$, the current through the device. The Josephson coupling $E_{\mathrm{J}}$ of the junctions

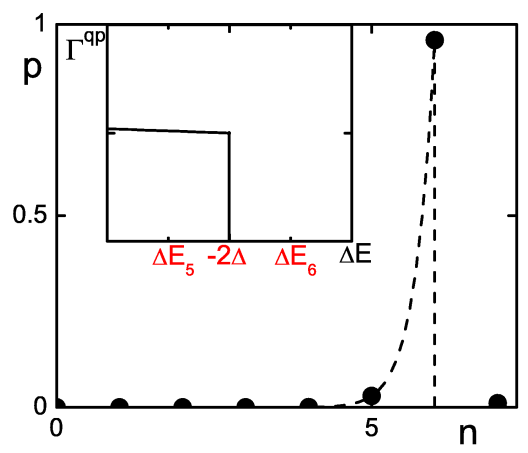

FIG. 1: A strongly squeezed distribution $p(n)$ of photon number states in a Josephson junction- $L-C$ oscillator driven by a SSET obtained for the parameters $\delta N_{\mathrm{G}}=-0.1, \mathrm{eV}=5.6$, $\Delta=2.2, g=0.01, \omega=0.4, \Omega=0.001, E_{\mathrm{J}}=0.1$ (all energies in units of $E_{C}$ ), and $\kappa / \gamma=0.004$. The inset shows the energy dependence of the quasiparticle transition rate and energy differences for two transitions, $\Delta E_{n}=E_{-, n+1}-E_{1, n}-e V$. Due to the anharmonicity of the oscillator they lie above and below the threshold.

should be small compared to the charging energy scale, $E_{C}=e^{2} / 2 C$ ( $C$ is the total capacitance of the island $)$, and the superconducting gap $\Delta$. It leads to coherent Cooper pair tunneling, and even in the considered limit, has pronounced consequences when two charge states differing by one Cooper-pair are nearly degenerate. In addition, quasiparticles can tunnel incoherently (with rate $\propto \gamma)$ when the energy difference between initial and final states is sufficient to create the quasiparticle excitation, i.e., when it exceeds twice the superconducting energy gap (assumed equal for electrodes and island), $|\Delta E| \geq 2 \Delta$. At the threshold tunneling sets in with a sharp step.

The SSET is tuned to the regime of the JQP cycle [17, 18, where the current is carried by a combination of Cooper pair transfer through one junction onto the is- 


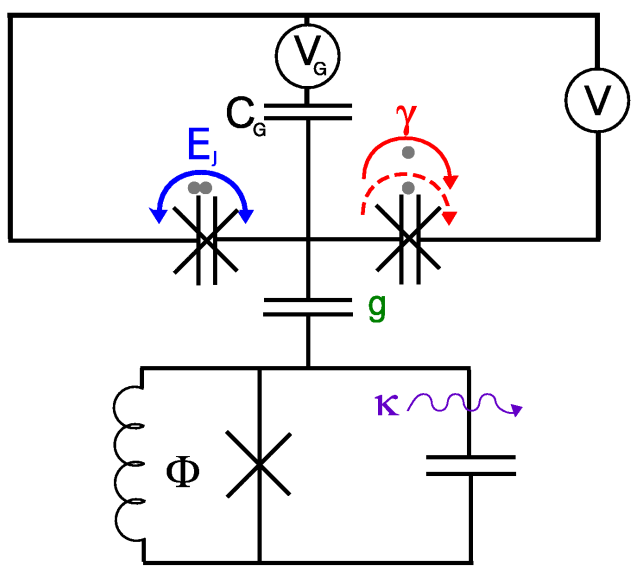

FIG. 2: A SSET with Josephson coupling $E_{\mathrm{J}}$ and quasiparticle tunneling rate proportional to $\gamma$, coupled with strength $g$ to an anharmonic oscillator. The oscillator's dissipation is characterized by the parameter $\kappa$. To get the right sign for the anharmonicity we use the flux $\Phi$. The current is determined by the transport voltage $\mathrm{V}$ and the gate voltage $V_{G}$ which is coupled to the island by the capacitance $C_{G}$.

land followed by two consecutive quasiparticle tunneling processes through the other junction. The energy for this process is provided by the voltage source. In oder to enhance the effect we consider in the following an asymmetric SSET, similar to those studied in Ref. 12, with one junction (where the Cooper pair is transferred during the JQP cycle) having a much stronger Josephson coupling than the other one.

The charge on the SSET is coupled capacitively, with strength characterized by $g$, to an anharmonic oscillator. This oscillator can be realized, e.g., by a circuit combining a capacitor, an inductor, and a non-linear element such as a Josephson junction, as shown in fig. 2. To get strong squeezing we need a positive quartic term in the potential energy, which can be achieved by shifting the minima of the inductive energy and Josephson coupling relative to each other by biasing the circuit with a half-integer number of flux quanta. Also nanomechanical oscillators must have an anharmonicity with positive sign (as studied, e.g., in Ref. [19]) for the phonon number squeezing mechanism described below to work.

The coherent dynamics of the coupled SSET and oscillator is described by the Hamiltonian

$$
\begin{aligned}
H_{0} & =E_{C}\left(N-N_{\mathrm{G}}\right)^{2}-E_{\mathrm{J}} \cos \left(\phi_{\mathrm{L}}\right) \\
& +g(N-1)\left(a^{\dagger}+a\right)+\omega a^{\dagger} a+\Omega\left(a^{\dagger} a\right)^{2} .
\end{aligned}
$$

The charging energy depends on the number of charges on the island, $N=N_{\mathrm{L}}-N_{\mathrm{R}}$, where $N_{\mathrm{L} / \mathrm{R}}$ are the numbers of charges which have tunnelled across the left/right junction and the gate charge $N_{G}=C_{G} V_{G}$. Cooper pair tunneling, assumed to dominate across the left junction, depends on the phase $\phi_{\mathrm{L}}$, which is conjugate to $N_{\mathrm{L}},\left[N_{\mathrm{L}}, e^{i \phi_{\mathrm{L}}}\right]=2 e^{i \phi_{\mathrm{L}}}$. The oscillator's eigenfrequency $\omega$ should be of the same order as the Josephson coupling $E_{\mathrm{J}}$. The anharmonicity $\Omega$ is weak, $\Omega\left\langle n^{2}\right\rangle \ll \omega\langle n\rangle$, where $\langle n\rangle$ is the average number of photons. The oscillator couples to the SSET with strength $g$. Without loss of generality we choose the oscillator to be at its equilibrium position for the island charge $N=1$.

In addition to the coherent dynamics, governed by $H_{0}$, the state of the system evolves due to incoherent quasiparticle tunneling in the SSET and due to dissipative processes in the oscillator. These effects will be described in the frame of a Liouville equation.

We tune the gate charge close to a symmetry point, $N_{\mathrm{G}} \approx-1$, such that only the charge states $N=0,1,2$ are of importance [20]. The two even states, $N=0,2$, are Josephson coupled to form the basis states

$$
\begin{aligned}
& |\uparrow\rangle=\cos \frac{\chi}{2}|N=0\rangle-\sin \frac{\chi}{2}|N=2\rangle, \\
& |\downarrow\rangle=\sin \frac{\chi}{2}|N=0\rangle+\cos \frac{\chi}{2}|N=2\rangle,
\end{aligned}
$$

with energies

$$
\epsilon_{\uparrow / \downarrow}=\left(1+\delta N_{\mathrm{G}}^{2}\right) E_{C} \pm \frac{1}{2} \sqrt{E_{\mathrm{J}}^{2}+16 \delta N_{\mathrm{G}}^{2} E_{C}^{2}} .
$$

Here $\tan \chi=E_{\mathrm{J}} / 4 \delta N_{\mathrm{G}} E_{C}$, and $\delta N_{\mathrm{G}}=N_{\mathrm{G}}-1$ is the deviation from the symmetry point. In addition we consider the odd state $|N=1\rangle$ with energy $\epsilon_{1}=E_{C} \delta N_{\mathrm{G}}^{2}$.

We assume that the system is operated in the regime of vacuum Rabi oscillations, where the energy difference of the SSET states $\delta \epsilon=\epsilon_{\uparrow}-\epsilon_{\downarrow}$ is close to the oscillator frequency $\omega$, and the SSET and photon number states $|n\rangle$ get strongly entangled. For weak detuning $\delta \omega=\omega-\delta \epsilon$

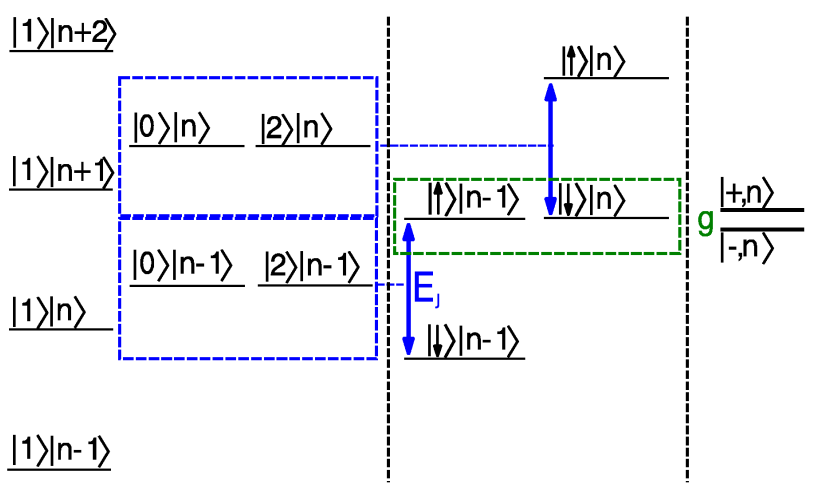

FIG. 3: The charge states $|N=0\rangle$ and $|N=2\rangle$ (here shown exactly at degeneracy) are coupled by the Josephson coupling $E_{\mathrm{J}}$ and form the basis states $|\uparrow\rangle$ and $|\downarrow\rangle$. Tuned to a resonance with the oscillator they form the eigenstates $| \pm, n\rangle$ of the coherent part of the Hamiltonian. Also the odd states $|1\rangle|n\rangle$ are shown. Their energy is shifted by an amount of the order of $E_{C}$ relative to the qubit states, but to fit into the plot this shift is not drawn to scale. 

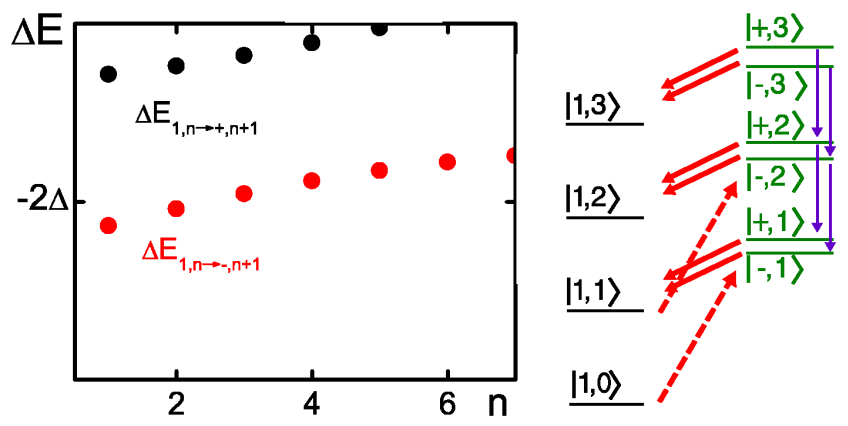

FIG. 4: The energy differences $\Delta E_{1, n \rightarrow \pm, n+1}$ as a function of photon number $n$ for the same parameters $\delta \omega, \Omega$ and $\bar{g}$ as used in fig. 1. Also shown is a cycle of transitions, which increases the number of photons in the oscillator. The dashed arrows correspond to the rate $\Gamma_{1, n \rightarrow \pm, n+1}^{\mathrm{qp}}$ and the solid arrows correspond to the rate $\Gamma_{ \pm, n \rightarrow 1, n}^{\mathrm{qp}}$. The vertical transitions are due to the dissipation decreasing the number of photons.

we can approximate the eigenstates of $H_{0}$ by

$$
\begin{aligned}
|+, n\rangle & =\sin \frac{\eta}{2}|\uparrow\rangle|n-1\rangle+\cos \frac{\eta}{2}|\downarrow\rangle|n\rangle, \\
|-, n\rangle & =\cos \frac{\eta}{2}|\uparrow\rangle|n-1\rangle-\sin \frac{\eta}{2}|\downarrow\rangle|n\rangle .
\end{aligned}
$$

The rotation angle, $\tan \eta=2 \bar{g} \sqrt{n} / \delta E(n)$, depends on the effective detuning, $\delta E(n)=\delta \omega+\Omega(2 n-1)$, and the effective coupling, $\bar{g}=g\langle\uparrow|(N-1)| \downarrow\rangle$. In addition, the states $|N=1\rangle|n\rangle$ with a single excess charge on the island are eigenstates of the system. The energies of the relevant states are then given by

$$
\begin{aligned}
E_{ \pm, n}= & \epsilon_{\uparrow}+E_{\mathrm{osc}}(n-1)+\frac{1}{2} \delta E(n) \\
& \pm \frac{1}{2} \sqrt{4 \bar{g}^{2} n+\delta E(n)^{2}} \\
E_{1, n}= & \epsilon_{1}+E_{\mathrm{osc}}(n),
\end{aligned}
$$

where $E_{\mathrm{osc}}(n)=\omega n+\Omega n^{2}$ is the energy of the anharmonic oscillator.

The Liouville equation for the reduced density matrix of the composite system accounts for dissipative processes. Assuming the transition rates to be weak compared to the energy spacings we can use the rotating wave approximation and neglect off-diagonal elements. The probability of the system to be in the state $|i\rangle$ is then determined by the simple master equation

$$
\dot{\rho}_{i}=\sum_{j}\left(\Gamma_{j \rightarrow i} \rho_{j}-\Gamma_{i \rightarrow j} \rho_{i}\right) .
$$

The transition rates $\Gamma_{i \rightarrow j}=\Gamma_{i \rightarrow j}^{\mathrm{qp}}+\Gamma_{i \rightarrow j}^{\kappa}$ account for quasiparticle tunneling and for transitions caused by the dissipation of the oscillator. Since both have been described earlier, e.g. in Refs. [16, 21], it is sufficient to quote the results.

The quasi-particle tunneling rate, assumed to dominate in the right junction, is given by

$$
\Gamma_{i \rightarrow j}^{\mathrm{qp}}=\left|\left\langle j\left|e^{i \phi_{\mathrm{R}} / 2}\right| i\right\rangle\right|^{2} I_{\mathrm{qp}}\left(\Delta E_{i \rightarrow j}\right) .
$$

The operator $e^{i \phi_{\mathrm{R}} / 2}$ transfers a single charge from the island to the right lead. (For the bias and low temperatures considered single charges tunnel only in this direction.) The relevant energy difference $\Delta E_{i \rightarrow j}=E_{j}-E_{i}-e V$ also includes the work provided by the voltage source. The strength and energy dependence of the rate are related to the quasiparticle tunneling current, $I_{\mathrm{qp}}(E)=$ $\gamma \int d E^{\prime} N\left(E^{\prime}\right) N\left(E^{\prime}-E\right) f\left(E^{\prime}\right)\left[1-f\left(E^{\prime}-E\right)\right]$, which depends on Fermi functions and the densities of states, $N(E)=\Theta(|E|-\Delta)|E| / \sqrt{E^{2}-\Delta^{2}}$. At low temperature we need to consider only inelastic transitions where energy is gained, i.e., $\Delta E_{i \rightarrow j}<0$. Due to the gap in the DOS the rate vanishes for $\Delta E_{i \rightarrow j}>-2 \Delta$, but jumps to a finite value if $\Delta E_{i \rightarrow j} \leq-2 \Delta$. For an anharmonic oscillator this energy difference depends on the photon number $n$. Hence at a certain value of $n$ the threshold may be passed, beyond which the transitions vanish abruptly (as shown in Fig. 1.) This cut-off is essential for the creation of a strongly squeezed photon distribution.

The dissipation in the oscillator introduces further transitions,

$$
\Gamma_{i \rightarrow j}^{\kappa}=\frac{\kappa|\langle j|a| i\rangle|^{2}}{\left|1-\exp \left(\left[E_{j}-E_{i}\right] / k T\right)\right|} .
$$

The parameter $\kappa$ summarizes all details of the oscillator's dissipation at the energy scale $E_{j}-E_{i}$. Most important are transitions with energy differences of the order of $\omega$. We consider low temperatures, where only relaxation processes occur.

If the transport voltage $V$ is large compared to the gap, the number of photons in the oscillator increases for positive $\delta N_{\mathrm{G}}$, until a balance between driving and dissipation - which is proportional to $n$ - is reached. The distribution of photon numbers is peaked around the average value $n_{\mathrm{av}} \propto \gamma / \kappa$, and the Fano factor $F=\left(\left\langle n^{2}\right\rangle-\langle n\rangle^{2}\right) /\langle n\rangle$ is slightly smaller than one. In this situation one observes some photon number squeezing [16, however it is weak and easily destroyed by temperature. For negative detuning $\delta N_{\mathrm{G}}$ the SSET, similar as an ac-driven qubit [11, can serve to cool the oscillator.

As the voltage is decreased certain rates can be pushed beyond the threshold at $\Delta E_{i \rightarrow j}=-2 \Delta$. The rates with the largest energy differences $\Delta E_{1, n \rightarrow \pm, n+1}$ will be cutoff first. These are given by

$$
\begin{aligned}
\Delta E_{1, n \rightarrow \pm, n+1}= & \epsilon_{\uparrow}-\epsilon_{1}-e V+\frac{1}{2} \delta E(n+1) \\
& \pm \frac{1}{2} \sqrt{4 \bar{g}^{2}(n+1)+[\delta E(n+1)]^{2}} .
\end{aligned}
$$

The upper branch will be cut-off first. The most interesting case is shown in fig. 4, where the cycle stops completely beyond a certain number of photons. We will now describe how to tune the system to reach this situation.

Strong squeezing requires that the energy difference $\Delta E_{1, n \rightarrow-, n+1}$ increases with increasing photon number $n$ (as is the case in fig. 44. The condition 

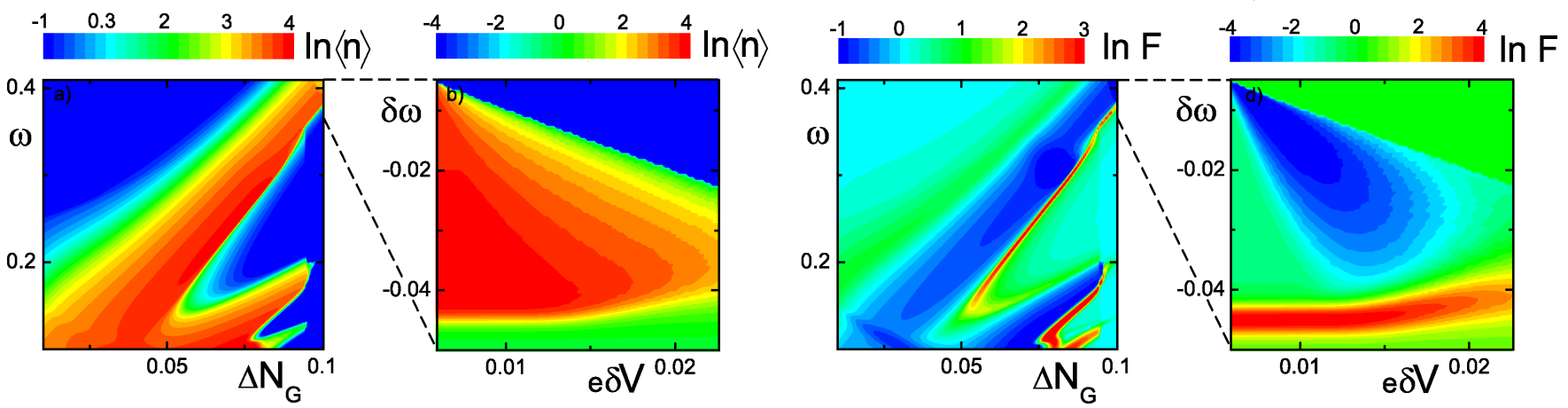

FIG. 5: (a) and (c): Average photon number $\langle n\rangle$ and Fano factor $F$ as a function of the oscillator frequency $\omega$ and the gate charge $\Delta N_{0}$, for $e V=5.62, g=0.01, \Omega=0.0005, E_{\mathrm{J}}=0.18, \kappa / \gamma=0.02$ (all energies in units of $\left.E_{C}\right)$. (b) and (d): Average photon number $\langle n\rangle$ and Fano factor $F$ as a function of the frequency detuning $\delta \omega$ and $e \delta V$ for $\Delta N_{0}=-0.1$.

$$
\begin{aligned}
\frac{\partial}{\partial n}\left(\Delta E_{1, n \rightarrow-, n+1}\right) & >0 \text { requires } \\
\Omega> & \frac{1}{2}\left(\delta \omega+\sqrt{2 \bar{g}^{2}+\delta \omega^{2}}\right) .
\end{aligned}
$$

This condition is independent of $n$, i.e., the energy difference either decreases or increases with $n$ for all states. We can see from eq. 10 that for a negative detuning, $\delta \omega<0$, an anharmonicity smaller than $\bar{g}$ is sufficient.

Squeezing can be observed below a certain threshold for the transport voltage. For large $n$ there is a limiting value for $\Delta E_{1, n \rightarrow-, n+1}$ which has to be larger than $-2 \Delta$; otherwise there is no point where the rate gets cut-off. From this we get a condition for the voltage

$$
\frac{1}{2}\left(\delta E(1)-\sqrt{4 \bar{g}^{2}+[\delta E(1)]^{2}}\right)<e \delta V<-\bar{g}^{2} / 2 \Omega,
$$

with $e \delta V=e V-2 \Delta+\epsilon_{1}-\epsilon_{\uparrow}$. The right hand inequality guarantees that there is a cut-off, and the left hand side guarantees that the cycle does not stop already at zero photons. If the conditions given by Eqs. 110 and 11) are met, the rates are cut-off at

$$
n_{\mathrm{cut}}=\frac{e \delta V(e \delta V-\delta \omega+\Omega)}{\bar{g}^{2}+2 e \delta V \Omega} .
$$

In order to have significant effects of the cut-off, we also have to require that $n_{\text {av }}>n_{\text {cut }}$.

We can optimize the system by choosing a negative detuning, $\delta \omega<0$, precisely in a way that the system is in resonance at the cut-off, $\delta E\left(n_{\text {cut }}\right)=0$. This means

$$
\delta \omega=\Omega\left[1-2(e \delta V)^{2} / \bar{g}^{2}\right] .
$$

In this case we get $n_{\text {cut }}=(e \delta V)^{2} / \bar{g}^{2}$.

We solved for the stationary distribution of eq. (6) in the product base of the charge states $N=0,1,2$ and many Fock states with $n \leq 200$ sufficient to guarantee convergence. In fig. 5 (a) and (c) we plot the average photon number and the Fano factor for a fixed transport voltage. The maxima in the photon number and the minima in the Fano factor correspond to $\omega=\delta \epsilon$ or to higher order resonances. The region where the conditions (10) and 111) are fulfilled lies in the center of fig 5 (d). Here we find strong squeezing.

In fig. 1 we show the probability distribution for the oscillator states $p(n)=\operatorname{Tr}(\rho|n\rangle\langle n|)$ for parameters which meet eqs. 10), 111) and (13). For these parameters the Fano factor is particularly small, $F \approx 0.01$, and one can clearly see the effect of the cut-off. The rates are cut at $n=7$. Therefore we have a sharp maximum in $p$ at $n=6$ and then a sudden drop. The probabilities above $n=6$ are not exactly zero, because the numerically calculated quantum states allow for more none-zero matrix elements in the transition rates (7) than the approximations given by eqs. (4). However, these additional rates are much smaller than $\Gamma_{1, n \rightarrow \pm, n+1}^{\mathrm{qp}}$ and $\Gamma_{ \pm, n \rightarrow 1, n}^{\mathrm{qp}}$, as can be seen by the significant drop in $p$ for $n>6$.

Conclusion. A strongly squeezed photon number distribution can be produced in a solid state anharmonic oscillator coupled to a SSET. It requires an oscillator with frequency in the $\mathrm{GHz}$ range, a positive quartic term, and sufficiently low dissipation, such that the inequalities $\omega>g>\gamma \gg \kappa$ are satisfied. For the example presented in fig. 5 a Q-factor of the order of $10^{4}$ is sufficient. Nanomechanical oscillators, with $Q$-Factors of the order of $10^{5}$ have been produced [22], but reaching the $\mathrm{GHz}$ range is difficult. Circuits formed of a Josephson junction and $L-C$ elements can satisfy the requirements and have the advantage of tunable anharmonicity and frequency, which in turn allows selecting the squeezed photon number state. Furthermore, these circuits can be coupled to superconducting qubits, which have been demonstrated to allow measuring the oscillator state as a state-dependent frequency shift 7 .

Acknowledgment: We thank V. Brosco, M. P. Blencowe, O. Astafiev, and Y. Nakamura for stimulating discussion. The work is part of the EU IST Project EuroSQIP. 
[1] N. Gisin, G. Ribordy, W. Tittel, and H. Zbinden, Rev. Mod. Phys. 74, 145 (2002).

[2] E. Knill, R. Laflamme, and G. J. Milburn, Nature 409, 46 (2001).

[3] V. Giovannetti, S. Lloyd, and L. Maccone, Science 306, 1330 (2004).

[4] B. T. H. Varcoe, S. Brattke, M. Weidinger, and H. Walther, Nature 403, 743 (2000).

[5] A. Wallraff, D. I. Schuster, A. Blais, L. Frunzio, R.S. Huang, J. Majer, S. Kumar, S. M. Girvin, and R. J. Schoelkopf, Nature 431, 162 (2004).

[6] I. Chiorescu, P. Bertet, K. Semba, Y. Nakamura, C. S. P. M. Harmans, and J. E. Mooij, Nature 431, 159 (2004).

[7] D. I. Schuster, A. Wallraff, A. Blais, L. Frunzio, R.S. Huang, J. Majer, S. M. Girvin, and R. J. Schoelkopf, Phys. Rev. Lett. 94, 123602 (2005).

[8] D. I. Schuster, A. A. Houck, J. A. Schreier, A. Wallraff, J. M. Gambetta, A. Blais, L. Frunzio, J. Majer, B. Johnson, M. H. Devoret, S. M. Girvin, and R. J. Schoelkopf, Nature 445, 515 (2007).

[9] A. Blais, R.-S. Huang, A. Wallraff, S. M. Girvin, and R. J. Schoelkopf, Phys. Rev. A 69, 062320 (2004).

[10] E. Il'ichev, N. Oukhanski, A. Izmalkov, Th. Wagner, M. Grajcar, H.-G. Wagner, A. Yu. Smirnov, A. M, van den Brink, M. H. S. Amin, and A. M. Zagoskin, Phys. Rev. Lett. 91, 097906 (2003).

[11] J. Hauss, A. Fedorov, C. Hutter, A. Shnirman, and G. Schön, Phys. Rev. Lett. 100, 037003 (2008).

[12] D. Astafiev, K. Inomata, A. O. Niskanen, T. Yamamoto, Yu. A. Pashkin, Y. Nakamura, and J. S. Tsai, Nature 449, 588 (2007).

[13] D. A. Rodrigues, and A. D. Armour, New J. Phys. 7, 251 (2005).

[14] M. P. Blencowe, J. Imbers, and A. D. Armour, New J. Phys. 7, 236 (2005).

[15] A. A. Clerk, and S. Bennet, New J. Phys, 7238 (2005).

[16] D. A. Rodrigues, J. Imbers, and A. D. Armour, Phys. Rev. Lett. 98, 067204 (2007).

[17] T. A. Fulton et. al., Phys. Rev. Lett. 63, 1307 (1989).

[18] A. Maassen van den Brink, G. Schön, and L.J. Geerligs, Phys. Rev. Lett. 67, 3030 (1991); A. Maassen van den Brink, A.A. Odintsov, P.A. Bobbert, and G. Schön, Z. Physik B - Condensed Matter 85, 459 (1991).

[19] R. Almog, S. Zaitsev, O. Shtempluck, and E. Bucks, Phys. Rev. Lett. 98, 078103 (2007).

[20] Y. Makhlin, G. Schön, and A. Shnirman, Rev. Mod. Phys. 73, 357 (2001).

[21] M.-S. Choi, F. Plastina, and R. Fazio, Phys. Rev. B. 67, 045105 (2003).

[22] A. Naik, O. Buu, M. D. LaHaye, A. D. Armour, A. A. Clerk, M. P. Blencowe, and K. C. Schwab, Nature 443, 193 (2006). 